\title{
DETERMINAÇÃO DOS PARÂMETROS CINÉTICOS DO PROCESSO DE DEGRADAÇÃO TÉRMICA DA ANTOCIANINA EXTRAÍDA DO REPOLHO ROXO
}

\author{
T. K. ANDRIOLI, K. C. V. DA SILVA, K. L. HOLEK, A.E. DA COSTA e J. K. \\ ANDREAZZA
}

UNISOCIESC - Joinville/SC, Faculdade de Engenharia Química

E-mail para contato: janaina.andreazza@sociesc.com.br

\begin{abstract}
RESUMO - Antocianinas são corantes encontrados em vegetais, responsáveis pela cor de folhas, flores e frutos. $O$ interesse que envolve os estudos cinéticos da antocianina retirada do repolho roxo se deve às perspectivas de uso desses pigmentos como corantes aplicados nos mais variados segmentos industriais, tais como o de alimentos, o de cosméticos e o farmacêutico. O presente trabalho teve como objetivo determinar os parâmetros cinéticos da degradação térmica das antocianinas extraídas do repolho roxo, nas temperaturas de 50,70 e $90^{\circ} \mathrm{C}$. A metodologia de análise foi dividida em duas fases, a primeira experimental e outra de simulação numérica e computacional baseada no modelo matemático fenomenológico do processo de degradação. Os resultados obtidos experimentalmente, demonstram um aumento na constante de degradação térmica $\left(k_{c}\right)$ e consequentemente uma redução da meia-vida $\left(t_{1 / 2}\right)$. Os resultados da otimização do modelo matemáticos também demonstram que a ordem da reação também aumenta com a temperatura. O modelo matemático fenomenológico é solucionado numericamente pelo método de Euler e implementado no software Matlab.
\end{abstract}

\section{INTRODUÇÃO}

O uso de corantes na indústria de alimentos é uma prática comum. Porém, há o problema que ocorre com alguns corantes alimentares, que além de não oferecerem nenhum valor nutritivo, podem causar reações tóxicas no metabolismo, ocasionando alergias, alterações no comportamento e carcinogenicidade. Desse modo, as indústrias estão investindo em pesquisas para modificar seus processos, substituindo os corantes artificiais de seus produtos por corantes naturais (FOOD BUSINES NEWS, 2015).

Levando em consideração o risco à saúde desses corantes artificiais, o corante natural antocianina é estudado como uma alternativa de substituição. Porém, tem como desvantagem a sua baixa estabilidade térmica, precisando ser monitorado após processamento para garantir uma melhor conservação do aspecto sensorial dos produtos diminuindo a sua degradação. Por isso, é necessário compreender seu processo de degradação, buscando formas de aumentar sua estabilidade (LOPES et al., 2007). 
Esse corante natural, antocianina, apresenta propriedades benéficas à saúde humana, além de propriedades farmacológicas, sendo utilizadas para fins terapêuticos. Já foram comprovados cientificamente seus efeitos anticarcinogênicos (HAGIWARA et al., 2001) também antioxidantes (WANG et al., 2000) e antivirais (KAPADIA et al., 1997).

Uma previsão do comportamento de degradação pode ser obtida por intermédio da simulação computacional, uma vez que esta é uma forma rápida, objetiva e econômica de determinar os perfis desejados em diferentes condições experimentais de operação.

Com objetivo de determinar a constante de degradação térmica e definir as funções termodinâmicas são realizados testes de degradação da antocianina em diferentes temperaturas, deduzido o modelo matemático fenomenológico, solucionado numericamente pelo método de Euler. A implementação computacional foi feita no software MatLab. O modelo matemático é validado comparando os dados experimentais com os simulados.

A aplicação de modelos para prever e interpretar parâmetros cinéticos, como a ordem e a taxa de reação, energia de ativação, entalpia, entropia e Gibbs, são essenciais para definir as trocas de qualidade que possam ocorrer ao longo do processamento térmico das antocianinas (AHMED et al., 2002; KARA; ERÇELEBI, 2013).

\section{MATERIAIS E MÉTODOS}

A investigação do problema foi realizada em duas fases. A primeira fase foi o levantamento de dados experimentais. Em seguida, na segunda fase realizou-se a dedução do modelo matemático fenomenológico do fenômeno de degradação das antocianinas do repolho roxo em batelada sob agitação discretizado pelo método de Euler. Após, implementou-se numericamente no software Matlab. Na sequencia o modelo foi validado com os dados experimentais e determinada as constantes cinéticas de degradação.

\subsection{Ensaios experimentais da extração e degradação da antocianina}

Para realizar os experimentos de degradação térmica da antocianina realizou-se a etapa de extração do corante, o qual a matéria prima utilizada foi o repolho roxo e como solvente usou-se o ácido acético $\left(\mathrm{CH}_{3} \mathrm{COOH}-20 \%\right)$ juntamente com água deionizada.

O repolho roxo foi desfolhado por completo desprezando apenas as folhas menores. Em seguida lavou-se cada uma das folhas com cautela e retirou-se os talos e as nervuras centrais do vegetal, picou-se as folhas em tamanhos de pequenas placas delgadas.

$\mathrm{Na}$ extração das antocianinas é utilizado soluções tampão de Mcllvaine, para a padronização do $\mathrm{pH}$ e diluição das amostras para posterior leitura no espectrofotômetro de UV visível. A solução tampão de Mcllvaine foi prepara com 15,83mL de ácido cítrico $0,1 \mathrm{M}$ juntamente com 4,1mL de fosfato dissódico 0,1M. Fechando um volume de $20 \mathrm{~mL}$ de solução tampão para a realização dos experimentos. De acordo com Xavier (2004), o pH adequado para realizar a leitura da absorbância das antocianinas se encontra entre 2,4 a 3,3. Portanto, adotou-se para este experimento um pH $3( \pm 0,3)$ para a leitura das amostras. O solvente de extração empregado foi o ácido acético à $20 \%$ e para auxílio na conservação das amostras utilizou-se 0,1g.L-1 de metabissulfito de sódio. 
Em 10 erlenmeyers colocou-se $15 \mathrm{~g}$ da amostra preparada de repolho roxo com $50 \mathrm{~mL}$ de solução de extração (relação MR/VS de 0,3). Conduziu-se as amostras até o equipamento shaker incubadora com $150,2 \mathrm{rpm}$ a temperatura de $27^{\circ} \mathrm{C}$. Ao término, as amostras foram centrifugadas por 5 min e reservadas em frasco âmbar até suas análises.

A cor da antocianina foi determinada por espectrometria e segundo Brouilliard, et al. (1993), as mesmas têm uma intensa absorção na faixa de 520 a $560 \mathrm{~nm}$ (região visível). Assim, a determinação da concentração de antocianinas nas amostras utilizou-se a lei de Lambert-Beer realizando uma curva de calibração tomando como padrão o corante sintético vermelho congo com comprimento máximo de onda em $500 \mathrm{~nm}$.

Logo, para os ensaios de degradação térmica da antocianina extraída do repolho roxo, foram utilizadas três temperaturas sendo 50,70 e $90^{\circ} \mathrm{C}$ de forma a obter a constante cinética (kc) para cada situação. Assim, preparou-se uma solução de $4 \mathrm{~mL}$ de corante contendo antocianinas e posteriormente colocou-se em frascos com tampa de rosca e submeteu-se em um banho termostático as amostras às temperaturas de estudo. Retirou-se as mesmas periodicamente até atingir a metade das concentrações. Os $\mathrm{pH}$ das alíquotas foram ajustados em $3( \pm 0,3)$ antes das análises no espectrofotômetro.

\subsection{Modelagem matemática fenomenológica}

O modelo matemático que descreve a degradação térmica da antocianina do repolho roxo com o tempo é baseado na lei de conservação da massa. Como hipóteses de trabalho, é considerado que o sistema é um taque perfeitamente agitado, as propriedades físicas e a temperatura são constantes e a cinética de degradação segue o modelo de potencia. Matematicamente, o modelo matemático é representado pelas equações 1 e 2 .

$$
\begin{gathered}
\frac{d C_{c}}{d t}=-k_{c} C_{c}^{n}, \\
C_{c}(0)=C_{c o}
\end{gathered}
$$

Onde Cc é a concentração de antocianina, $\mathrm{mg} \cdot \mathrm{m}^{-3}$, Cco é a concentração inicial de antocianina, mg. $\mathrm{m}^{-3}$, kc é a constante cinética de degradação, $\left(\mathrm{m}^{3} / \mathrm{mg}\right)^{\mathrm{n}-1} \cdot \mathrm{h}^{-1}$ e n é a ordem da reação.

Em se tratando de um problema de valor inicial, foi utilizado o método de Euler e implementado no software Matlab 2014a (licença UNISOCIESC, número 965574).

\section{RESULTADOS E DISCUSSÃO}

O método empregado para a análise dos dados cinéticos, tendo em vista a identificação da lei de velocidade da reação de degradação, foi o de meias-vidas. Os experimentos foram realizados até que a concentração inicial de antocianina reduz-se a metade, ou seja, atingissem o tempo de meia vida $\left(\mathrm{t}_{1 / 2}\right)$ para cada temperatura estudada. Na Figura 1 são apresentados os gráficos para as temperaturas de 50,70 e $90^{\circ} \mathrm{C}$. Pode-se observar que as antocianinas 
degradaram-se mais rapidamente com o aumento da temperatura, ocorrendo consequentemente o aumento dos valores de $k_{\mathrm{c}}$.

Figura 1 - Degradação de antocianinas extraída do repolho roxo para 50,70 e $90^{\circ} \mathrm{C}$

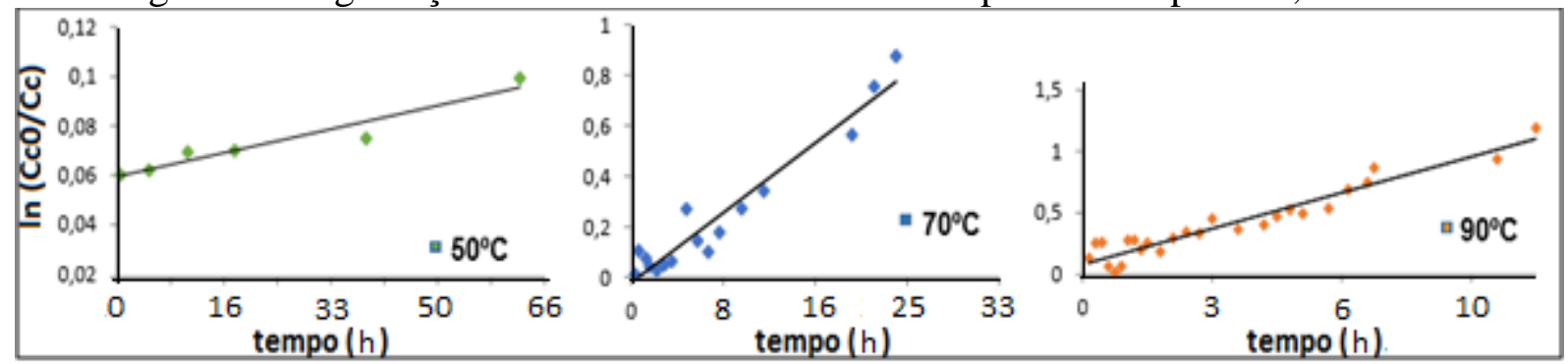

Em relação ao $t_{1 / 2}$ houve um aumento com o decréscimo da temperatura. Os valores da Cco das soluções para cada uma das temperaturas estudadas, os $\mathrm{t}_{1 / 2}$ e os valores $k_{\mathrm{c}}$ são mostrados na Tabela 1.

Tabela 1 - Valores Cco, $\mathrm{t}_{1 / 2}, k_{\mathrm{c}}$ e $\mathrm{R}^{2}$ para as temperaturas estudadas

\begin{tabular}{|c|c|c|c|c|}
\hline $\mathrm{T}\left({ }^{\circ} \mathrm{C}\right)$ & $\mathrm{Cco}\left(\mathrm{mg} / \mathrm{m}^{3}\right)$ & $\mathrm{t}_{1 / 2}(\mathrm{~h})$ & $k_{\mathrm{c}}\left(\left(\mathrm{m}^{3} / \mathrm{mg}\right)^{\mathrm{n}-1} \cdot \mathrm{h}^{-1}\right)$ & $\mathrm{R}^{2}$ \\
\hline 50 & 80,12 & 36,32 & 0,0191 & 0,9265 \\
\hline 70 & 76,08 & 27,04 & 0,0260 & 0,9331 \\
\hline 90 & 113,88 & 18,66 & 0,0371 & 0,9525 \\
\hline
\end{tabular}

A dependência da constante cinética da degradação térmica da temperatura segue a lei de Arrhenius. A Equação 3 traz a linearização da lei de Arrhenius, com essa equação são determinados o fator de frequência, ko, e a energia de ativação da reação de degradação $(\mathrm{E})$, sendo seus valores respectivamente de $1,0310.10^{4} \mathrm{~h}^{-1}$ e $63,081 \mathrm{~kJ} \cdot \mathrm{mol}^{-1}$.

$$
\ln k_{c}=17,537-16,639 \frac{1}{T}
$$

A $k_{c}$ e a E são utilizadas para calcular os valores da variação da entalpia $(\Delta \mathrm{H})$ nas temperaturas de 50,70 e $90^{\circ} \mathrm{C}$, obtendo os valores respectivos de $60,39 \mathrm{~kJ} / \mathrm{mol}, 60,23 \mathrm{~kJ} / \mathrm{mol}$ e $60,06 \mathrm{~kJ} / \mathrm{mol}$. Verifica-se que ocorre diminuição nos valores de $\Delta \mathrm{H}$ com o aumento da temperatura. Isto demonstra que a energia a ser quebrada para chegar ao estado de transição é semelhante nas temperaturas analisadas e diminui com o aumento da temperatura. Mercali et al. (2015), no processo de degradação das antocianinas do extrato de acerola e Mercali et al. (2013) no mesmo processo de degradação em antocianinas do extrato de jabuticaba, observaram os mesmos padrões para a entalpia.

A energia livre de Gibbs para a degradação das antocianinas teve propensão a um aumento sutil com o aumento da temperatura em estudos e o sinal positivo para $\Delta \mathrm{G}$ evidenciou a não espontaneidade da reação. Desse modo, para todas as temperaturas utilizadas no estudo da antocianina a reação de degradação não foi espontânea nas temperaturas de 50,70 e $90^{\circ} \mathrm{C}$. Mercali et al. (2013) obtiveram o mesmo padrão na degradação das antocianinas da polpa de acerola, com aquecimento entre 75 e $90^{\circ} \mathrm{C}$. 
Como já descrito anteriormente, os experimentos foram realizadas até atingir o $t_{1 / 2}$ nas temperaturas de 50,70 e $90^{\circ} \mathrm{C}$. Para a simulação computacional optou-se por um tempo maior, $120 \mathrm{~h}$, a fim de verificar o comportamento do perfil dinâmico da antocianina com tempo superior ao de meia vida. O parâmetro livre no modelo matemático é a ordem da reação da degradação da antocianina, n. Para sua obtenção, o modelo matemático é otimizado através da comparação dos seus resultados de concentração de antocianina simulados $\left(C c_{\text {sim }}\right)$ com os valores obtidos experimentalmente $\left(C c_{\text {exp }}\right)$. Para a estimativa do parâmetro $\mathrm{n}$ é utilizado o método dos mínimos quadrados, como mostra a Equação (4). Na Figura 2 é mostrado que o valor ótimo do parâmetro n, ou seja, onde o Erro é zero, para $50^{\circ} \mathrm{C}, 70^{\circ} \mathrm{C}$ e $90^{\circ} \mathrm{C}$ é respectivamente de $0,7,0,98$ e 1,16 .

$$
\text { Erro }=\left(C c_{\text {exp }}-C c_{\text {sim }}\right)^{2}
$$

Figura 2 - Otimização dos valores do parâmetro n para $50^{\circ} \mathrm{C}, 70^{\circ} \mathrm{C}$ e $90^{\circ} \mathrm{C}$.

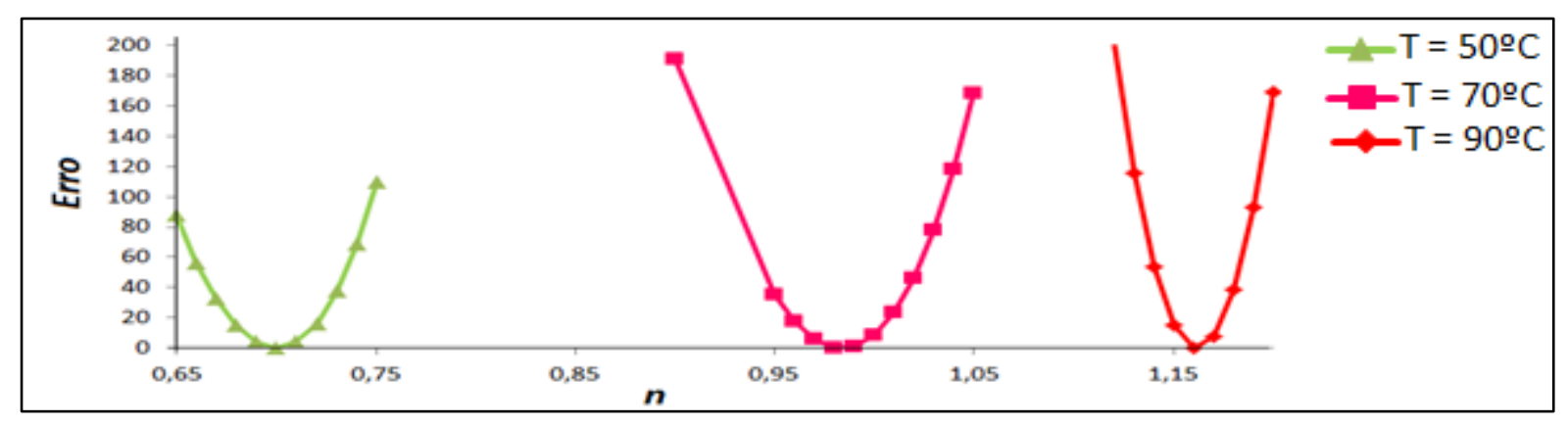

Figura 3 - Perfil da concentração de Antocianina para temperatura: (a) $50^{\circ} \mathrm{C}$, (b) $70^{\circ} \mathrm{C}$, (c) $90^{\circ} \mathrm{C}$ e (d) Influência da temperatura na degradação com o tempo.
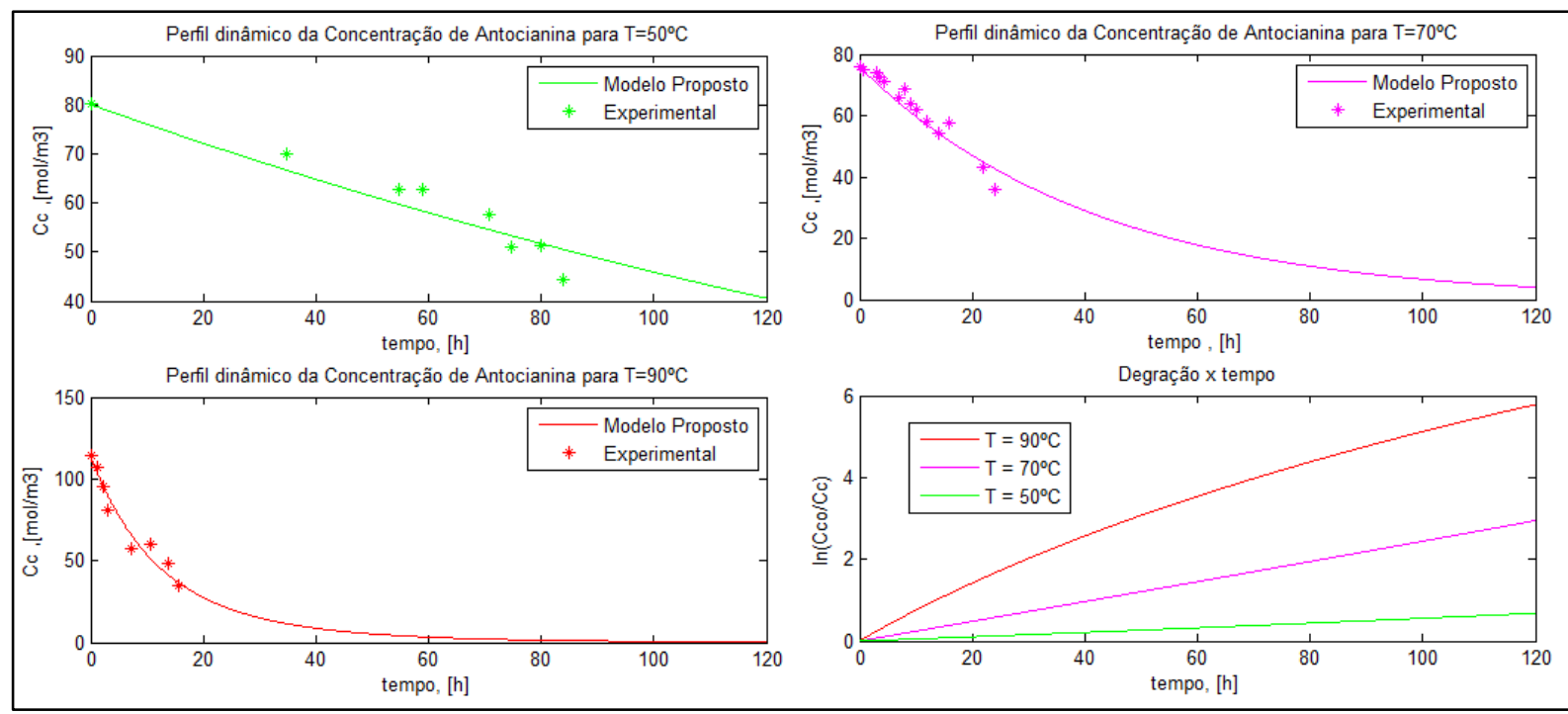

A Figura 3 ((a), (b) e (c)) apresenta os pontos experimentais obtidos até $t_{1 / 2}$ e o perfil dinâmico da concentração de antocianina simulado computacionalmente com a ordem de reação estimada de $0,68,0,98$ e 1,16 para as temperaturas de $50^{\circ} \mathrm{C}, 70^{\circ} \mathrm{C}$ e $90^{\circ} \mathrm{C}$ respectivamente. Na Figura 2 (d) são apresentados os perfis da evolução da degradação da 
antocianina em função do tempo, é nítido que com o aumento da temperatura também aumenta a velocidade de degradação da antocianina. Com este tempo de simulação, somente na temperatura de $50^{\circ} \mathrm{C}$ a degradação térmica estabilizou-se.

\section{CONCLUSÃO}

Por meio da pesquisa experimental e da simulação computacional foi possível prever o comportamento da degradação térmica das antocianinas do repolho roxo, constatando que a taxa de degradação de antocianinas aumenta à medida que a temperatura aumenta. Das análises experimentais foi possível caracterizar a reação de degradação das antocianinas como endotérmica e não espontânea, verificando que a variação negativa da entropia evidenciou que o estado de transição das moléculas de antocianinas é mais organizado estruturalmente. A ordem, a constante cinética de degradação das antocianinas do repolho roxo e os tempos de meia-vida são respectivamente $0,68,0,0191\left(\left(\mathrm{~m}^{3} / \mathrm{mg}\right)^{\mathrm{n}-1} \cdot \mathrm{h}^{-1}\right.$ e $36,32 \mathrm{~h}$ para $50^{\circ} \mathrm{C}, 0,98,0,0260$ $\left(\left(\mathrm{m}^{3} / \mathrm{mg}\right)^{\mathrm{n}-1} \cdot \mathrm{h}^{-1}\right.$ e $27,04 \mathrm{~h}$ para $70^{\circ} \mathrm{C}$ e $1,16,0,0371\left(\left(\mathrm{~m}^{3} / \mathrm{mg}\right)^{\mathrm{n}-1} \cdot \mathrm{h}^{-1}\right.$ e $18,66 \mathrm{~h}$ para $90^{\circ} \mathrm{C}$.

\section{REFERÊNCIAS}

FOOD BUSINESS NEWS. Mondelez to remove artificial colors, flavors by 2020. Foodbusinessnews.net.2015

HAGIWARA, Akihiro; Pronounced inhibition by a natural anthocyanin, purple corn color, of 2-amino-16-phenylimidazol $(4,5-b)$ pyridine (PhIP)-associated colorectal carcinogenesis in male F344 rats pretreated with 1,2-dimethylhydrazine. Cancer Letters, v. 171, n. 1, p. 17-25, agosto. 2001.

KAPADIA, G.J.; BALASUBRAMANIAN, V.; TOKUDA, H.I.; WASHINA, A.; NISHINO, $\mathrm{H}$. Inhibition of 12 Otetradecanoylphorbol- 13-acetate induced Epstein virus early antigen activation by natural colorants. Cancer Letters, Oxford, v.115, n.2, p.173-178. 1997.

LOPES Toni Jeffeson; XAVIER Marcelo Fonseca; QUADRI Mara Gabriela Novy; QUADRI Marintho Bastos. Antocianinas: uma breve revisão das características estruturais e da estabilidade. R. Bras. Agrociência, Pelotas, v.13, n.3, p. 291-297, jul./set. 2007.

MERCALI, G. D. et al. Evaluation of non-thermal effects of electricity on anthocyanin degradation during ohmic heating of jabuticaba (Myrciaria cauliflora) juice. Food Chemistry, v.171, p.200-206, 2015.

MERCALI, G. D. et al. Degradation kinetics of anthocyanins in acerola pulp: Comparison between ohmin and conventional heat treatment. Food Chemistry, v.136, p.853-857, 2013.

WANG, C.J. Protective effect of Hibiscus anthocyanins against tert-butyl hidroperoxideinduced hepatic toxicity in rats. Food and Chemical Toxicology, Oxford, v.38, n.5, p.411-416, 2000.

XAVIER, M. F. Estudo da Extração de Antocianinas em Colunas Recheadas. Florianópolis: UFSC, 2004. 120 p. Dissertação (Mestre em Engenharia Química) Universidade Federal de Santa Catarina, Florianópolis, 2004. 